\title{
Assessment of knowledge integration in student learning of momentum
}

\author{
Wangyi Xu๑, ${ }^{1,2}$ Qiaoyi Liu, ${ }^{2}$ Kathleen Koenig $\odot,{ }^{3}$ Joseph Fritchman, ${ }^{2}$ \\ Jing Han, ${ }^{2}$ Sudong Pan $\odot,{ }^{1, \dagger}$ and Lei Bao $\odot^{2, *}$ \\ ${ }^{1}$ East China Normal University, Faculty of Education, Shanghai 200062, China \\ ${ }^{2}$ The Ohio State University, Department of Physics, Columbus, Ohio 43210, USA \\ ${ }^{3}$ University of Cincinnati, Department of Physics, Cincinnati, Ohio 45220, USA
}

(Received 1 January 2020; accepted 8 May 2020; published 26 May 2020)

\begin{abstract}
Momentum is a foundational concept in physics. Although it is often taught in introductory mechanics courses, there are a limited number of studies on this topic in the literature. The results from these studies have consistently shown that students have difficulties in understanding momentum, especially the connections between net force, time, and change in momentum, which are signs of fragmented knowledge structure and poor knowledge integration. This study adopts the conceptual framework representation to model student understanding and guide the design of an assessment test on momentum. Informed by the previous work on knowledge integration, the conceptual framework maps out the key concepts and their connections within a student's knowledge structure. Recent studies have shown that a conceptual framework can be used as a guide to create assessment items that follow different reasoning pathways, probing various misconceptions and student difficulties. In this study, an assessment of momentum was developed and tested among a large number of U.S. college freshmen and Chinese high school students. Based on testing and interview results, students' understanding is separated into three progression levels of knowledge integration including novicelike, transitional, and expertlike. Furthermore, the comparison between the two countries' curriculum and momentum test results indicates that an emphasis on the central idea of impulse-momentum theorem can be an essential instructional strategy to help students make the necessary connections within their knowledge structure, leading to a deeper conceptual understanding of momentum.
\end{abstract}

DOI: 10.1103/PhysRevPhysEducRes.16.010130

\section{INTRODUCTION}

In science education, it has been a concern for years that many students fail to develop deep understanding of essential scientific concepts after traditional instruction [1-5]. Students may show fluency in typical textbook problems, but when faced with problems that are context rich and less familiar, they tend to rely on pattern matching of memorized equations with little engagement of applying conceptual understanding $[1-4,6]$. When it comes to differentiating experts from novices, context dependence and connectedness of students' knowledge structures, as demonstrated in previous studies, are considered to be key factors [7-13]. Novices' fragmented knowledge structures

\footnotetext{
*Corresponding author. bao.15@osu.edu

Corresponding author. sdpan@phy.ecnu.edu.cn

Published by the American Physical Society under the terms of the Creative Commons Attribution 4.0 International license. Further distribution of this work must maintain attribution to the author(s) and the published article's title, journal citation, and DOI.
}

tend to be poorly clustered with few localized links that are mostly situated in familiar contexts [7,8,10-13], which lead to problem solving strategies that rely on memorized processes cued by surface features $[7,8]$. On the other hand, experts appear to have an integrated knowledge structure that is hierarchically arranged as a network around a few core principles. The structures involve many consistent and long-range links across all elements from concrete surface features to deep into the abstract domain [7,8,10-13]. This better enables experts to apply these principles across different domains and in unfamiliar contexts $[7,9]$.

When students successfully transition from novices to experts, they gain new and different ideas and build connections among them to form a more global, cohesive knowledge structure, a process commonly studied in the perspective of knowledge integration [14-18]. According to the theory of knowledge integration, in the process of establishing organization in a student's knowledge structure, a central idea provides an anchor point that connects other ideas, which serves as a central node to a hierarchically organized knowledge structure [14-17]. Experts, on the other hand, can prompt the central idea through a wide range of contexts when solving problems [14-17]. 
Informed by a large body of existing learning theories, particularly theories of conceptual change and knowledge integration, the conceptual framework representation has been developed $[19,20]$. This framework provides an operational tool to explicitly model students' knowledge structures that give rise to their conceptions, from novice to expert. In a conceptual framework, a learner's ideas are activated by and depend on contextual features. An expert would link the activated ideas and related conceptual components to form reasoning pathways around the central idea as the core anchor for forming a fully integrated knowledge structure. On the other hand, a novice often bypasses the central idea and develops direct links that stem from memorized algorithms or equations among surface features of a problem context. Even though the novice's approach may produce the correct results in some limited cases, it will quickly become unworkable as the number of variables and the complexity of the context increases. In comparison, associating a wide range of situations to the central idea will help learners form a well-integrated knowledge structure that supports the establishment of deep expertlike conceptual understanding.

Building a conceptual framework for a particular concept starts with identifying the central idea of that concept, along with contextual variables and relations relevant to that concept. Subsequently, the conceptual pathways can be built among the central idea and other elements related to the concept. Once a conceptual framework on a specific topic is established, it can be employed to develop assessment instruments that more accurately target students' knowledge structures and explicitly map out their status of conceptual understanding. In each of two recent studies $[19,20]$, a conceptual framework based on a physical principle (force motion [19], and light interference [20]) has been developed to map out the key concepts and their connections within a student's knowledge structure. The framework was then used as a guide to create assessment items that follow different pathways, probing various misconceptions and student difficulties. The assessments feature a mix of typical and atypical questions throughout as a means of quantifying the extent for which students are able to transfer the key physical principle to different contexts. It has been shown that with the aid of an underlying conceptual framework, the instruments can reveal students' reasoning and map out links and pathways contained in their knowledge structures. In addition, the results were shown to be in agreement with observations of students' problem solving behavior during interviews $[19,20]$.

Moreover, a topic-specific conceptual framework can be applied to design teaching interventions that aim to help students develop the missing links in their knowledge structures. Such interventions have been shown to be crucial to forming an integrated knowledge structure and achieving deep conceptual understanding [18]. In the case of force and motion, the new instruction intervention emphasizes the central idea and its applications in problem solving. The instructional approach is designed to explicitly introduce the central idea, while showing its application in problem solving with demonstrations and example problems. This new method of instruction has been shown to be effective in building connections within students' knowledge structures and promoting knowledge integration [19].

Following the endeavor to further explore the utility of the conceptual framework modeling approach with other physics topics, this study focuses on the topic of linear momentum (momentum for brevity from here forward). Momentum is a foundational concept in physics, which is often taught in an introductory mechanics course. However, there are a limited number of related studies, which have primarily illustrated that students have difficulties in understanding momentum and in applying it during problem solving [21-25]. In particular, a large portion of students have been shown to miss the connections between net force, time, and change in momentum [22]. This phenomenon has been attributed to the difficulty of visualization [22], or the incomplete explanation presented in textbooks and lectures [26]. Building upon the previous work, this study uses a conceptual framework representation in the context of momentum to design an assessment that tests students' conceptual understanding of momentum, while examining its viability. The goals of this study are as follows:

- Establish a conceptual framework model for the topic of momentum and apply the conceptual framework to analyze student difficulties in learning momentum through the knowledge integration perspective.

- Employ an assessment approach involving atypical (unfamiliar) contexts to probe students' levels of deep understanding and make inferences on their knowledge structures.

\section{METHODS AND DESIGN}

\section{A. The conceptual framework of momentum}

In this study, a conceptual framework was established to analyze student difficulties on momentum. Following previous studies $[19,20]$, when building a conceptual framework the first step is to identify the central idea of a concept, which provides the core explanatory mechanisms or premises for establishing the causal relations underpinning the concept. For momentum, the impulsemomentum theorem is chosen as the central idea for establishing the conceptual framework in this study [27]. It is worth noting that the choice of a central idea and the definition of a conceptual framework are mostly based on experts' views of physics and can have multiple forms and versions depending on the purpose and emphasis.

The impulse-momentum theorem states that the change in momentum of an isolated system is equal to the impulse of the net force acting upon it, which can be found by 
integrating the net force over the time period that the net force is applied. In this study, the students were enrolled in introductory level courses, which only considered constant forces. Any nonconstant forces were approximated by constant average forces. Therefore, only the case involving constant forces is considered in this study. In addition, this study focuses on the initial establishment of the concept of momentum, and therefore, only one-dimensional situations are considered. For a constant one-dimensional net force $F_{\text {net }}$ applied over a time period of $\Delta t$, the change in momentum of the system is given by $\Delta p=F_{\text {net }} \Delta t$. The impulse-momentum theorem can be used to describe the momentum changes due to force interactions between two objects (or systems). For a single object (or a system), the total change in its momentum is the sum of all the momenta changes resulting from all individual interactions. This can also be viewed as the total momentum change caused by the net force.

In the case where the system is isolated, only internal interactions exist with forces that obey Newton's third law. The isolated system experiences no net external force that alters its momentum, i.e., $F_{\text {net }}=0$. Therefore, by the impulse-momentum theorem, there is no change in momentum, which means that the total momentum of an isolated system is constant (or conserved). This result is known as momentum conservation, which is one of the most fundamental principles of physics. On the other hand, in the case where the system is not isolated, the momentum of the system is not conserved, and the change in momentum can be calculated using the impulse-momentum theorem.

Putting all these together, when solving problems that involve momentum, it is essential to identify whether the system is isolated, i.e., whether the sum of external forces is zero or not. The impulse-momentum theorem can then be applied, forming the central idea that conceptualizes specific problem-solving strategies.

Singh et al. demonstrated that students lack a coherent understanding of the momentum concept, and have difficulty applying it in different physical situations. They found that students often focus on the surface features of the problem and are distracted by irrelevant details [21]. A possible factor for this behavior is that in many courses students primarily encounter the typical momentum conservation problems, such as collisions within isolated systems. This may lead some students to develop problem-solving strategies that only apply the momentum conservation approaches, which bypass the essential connection between the change in momentum and net force applied over time. These students likely think of momentum only as a mathematical construct of $p=m v$, with little understanding of the conceptual underpinning and how it connects to other physical quantities [22]. For example, students often lack an understanding of the connection between impulse and change of momentum, which leads to difficulties in analyzing systems that are not isolated [22].
Consequently, questions involving impulse-momentum theorem are typically perceived to be more difficult than those involving the mere application of $p=m v$ in momentum conservation contexts $[21,22]$.

After a careful examination of the physics content and research literature on students' difficulties in understanding momentum, a conceptual framework containing the central idea and different reasoning pathways was developed as shown in Fig. 1. The box at the top contains the central idea, which includes the impulse-momentum theorem. When solving problems that involve momentum, experts often begin by analyzing all forces acting upon the system, possibly drawing free body diagrams, to see whether the net external force is zero. If this net force is zero, then the system is isolated and the impulse-momentum theorem leads to momentum conservation. If the net force is nonzero, then the system is not isolated and it is necessary to use the impulse-momentum theorem to relate the change in momentum $(m \Delta v)$ and the net external force $(F \Delta t)$. The central idea is also closely tied to a few other important concepts in Newtonian mechanics, such as Newton's second and third laws, which can be useful in problem solving as well. The middle layer of the framework consists of intermediate outcomes that arise from reasoning and other mathematical and logical manipulation processes. The bottom layer involves contextual features and variables, which include most of the quantities that directly relate to the given problem at a surface level, such as velocity, mass, force, and time. For most problems involving momentum, the task goal is often to calculate the velocity or the momentum of concerned objects. These outcome-related components are shown as "task goals" in Fig. 1.

The arrows linking the different contextual, conceptual, and outcome components represent the possible conceptual pathways of experts and novices. The solid arrows represent experts' conceptual pathways; while the possible pathways of novices are illustrated with dashed line arrows. For experts, the central idea serves as a central node to the connections, forming an integrated knowledge structure. Therefore, when solving momentum related problems, experts activate the central idea as guidance in their analysis to identify relevant quantities and problem-solving approaches. On the other hand, novices tend to make weak, local connections between the different layers, forming fragmented knowledge structures. These usually arise from rote memory or matching contextual variables with equations without deep understanding. In the case of momentum, students are often exposed to situations where the momentum of the system is conserved, which can be solved using the momentum conservation equations. However, novices, who lack an understanding of the central idea, often attempt to apply the momentum conservation equations for situations involving nonisolated systems, leading to difficulties and confusion in solving impulse related problems [21,22]. 


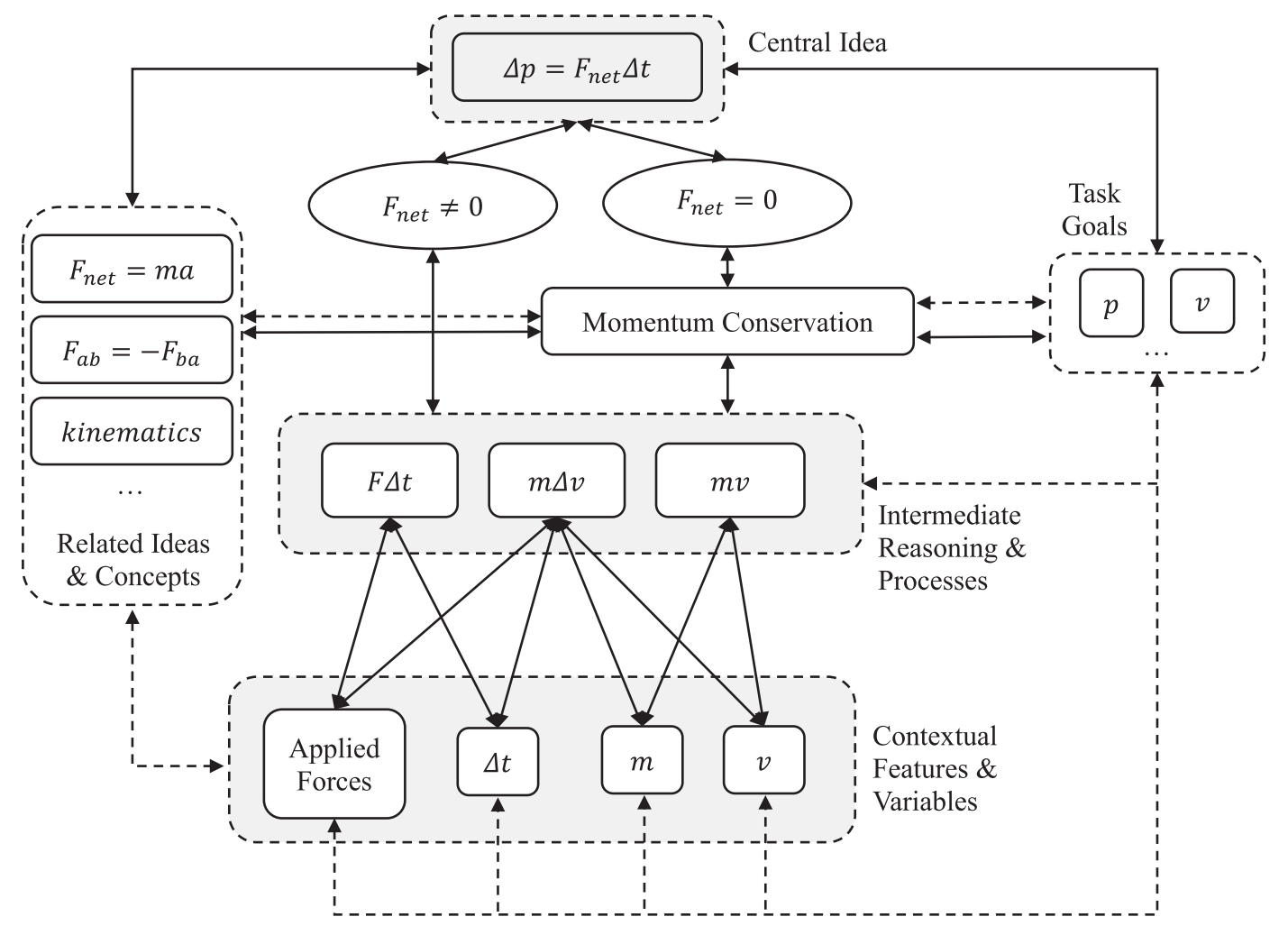

FIG. 1. Conceptual framework of momentum. Solid arrows represent experts' conceptual pathway, while the dashed lines represent the direct links between contextual features and the task outcomes that novices often make.

The connections shown in Fig. 1 are represented with double-headed arrows, indicating that a connection can be initiated from either side; however, it is possible for individual students to have only developed one direction of link activation among some of the connections. These students can be considered at intermediate levels of conceptual development.

\section{B. Modeling student understanding using the conceptual framework}

To help understand how the conceptual framework manifests itself within students' knowledge structures, students' conceptual understanding and problem-solving behaviors can be summarized into three developmental levels, which are explained using the representative pathways of the conceptual framework:

(i) Novice level: Students at this level have mostly fragmented knowledge structures and are able to only solve problems isomorphic to memorized examples and with direct applications of equations. For example, students tend to mechanically calculate the momenta using $p=m v$ with given velocities and masses. They are confused when a problem does not provide any velocities or masses. The conceptual pathways of these students are often present as local direct links between the contextual variables, equations, and task outcomes (see the dashed links shown in Fig. 1).

(ii) Intermediate-mixed level: Students at this level can engage in a deeper level of reasoning based on the contextual variables over the novice students; however, they still tend to rely on memorized examples and equations to aid their problem solving. The knowledge structures of these students have more developed links with emerging networklike constructs that to some extent overlap with the experts' network of connections. Students at this level often do well in solving the typical collision problems by applying momentum conservation equations, and can sometimes figure out the intermediate variables such as impulse and change of momentum when in familiar contexts. However, they often fail to reason through problems involving unfamiliar contexts, such as when variables and task goals are less obviously matched and require a more integrated understanding of the central idea in order to develop an effective problem-solving approach.

(iii) Expert level: Students at this level have developed a well networked expertlike knowledge structure. This allows them to relate contextual variables to the central idea, along with many of the intermediate processes and related concepts, to form a comprehensive package of resources to address the problem 
task. These students have a profound understanding of the conceptual components and the connections between them, and therefore can solve many questions across contexts.

Based on the differences of problem-solving strategies used by students in solving typical and atypical problems, one can make inference on the extent to which students' knowledge structures are fragmented or integrated. In this study, the established conceptual framework and the description of different levels of students' understanding will be used to guide the development of an assessment to evaluate the features of students' knowledge structures. Quantitative data will be collected to analyze the general categories of students' knowledge structures. In addition, follow-up interviews will be conducted to further examine students' thought processes and reasoning pathways. These results will be used to validate the conceptual framework of momentum.

\section{Design of the assessment}

Based on the conceptual framework of momentum, a test containing 11 multiple-choice questions was developed to assess students' understanding of momentum and Newton's third law. The test was designed based on questions used in instruction and previous research on the related topics [28]. Newton's third law is included because it is a key conceptual element connecting the impulse-momentum theorem to momentum conservation (see the conceptual framework in Fig. 1). To obtain an integrated understanding of momentum, which requires well-established connections between the impulse-momentum theorem and momentum conservation, it is critical for students to have a concrete conceptual understanding of Newton's third law. Therefore, three questions on Newton's third law were included in the test to assess students' understanding on this concept.

The questions were designed with both typical (familiar) and atypical (unfamiliar) contexts. The collision questions are considered typical as the contexts are commonly included in instruction as part of example problems or in-class demonstrations involving momentum conservation and Newton's third law. On the other hand, the impulsemomentum theorem is often applied in noncollision contexts, which are not often considered in the typical U.S. curriculum, and therefore, are less familiar (atypical). A summary of the question designs are provided in Table I and discussed below:

- Momentum conservation questions in typical collision context: Students are asked to identify the final velocity of the object after a collision, which is often solved by using the defining expression $p=m v$ along with momentum conservation, $\sum m_{i} v_{i}=\sum m_{f} v_{f}$.

- Impulse-momentum theorem questions in atypical noncollision context: Noncollision problems are considered atypical questions for U.S. students. Here, students are asked to identify the final momentum of
TABLE I. Assessment items used in this study.

\begin{tabular}{|c|c|c|}
\hline Concept domain & Context & Questions \\
\hline $\begin{array}{l}\text { Momentum } \\
\text { conservation (MC) }\end{array}$ & Collision & Q4, Q9, Q10 \\
\hline $\begin{array}{l}\text { Impulse-momentum } \\
\text { theorem (IM) }\end{array}$ & $\begin{array}{l}\text { Nonzero net force } \\
\text { Friction } \\
\text { Explosion }\end{array}$ & $\begin{array}{l}\text { Q2, Q8 } \\
\text { Q5, Q6 } \\
\text { Q7 }\end{array}$ \\
\hline Newton's third law (N3) & Collision & Q1, Q3, Q11 \\
\hline
\end{tabular}

an object with a nonzero net force applied for a duration of time. This type of problem can be solved by using the central idea, the impulse-momentum theorem, $\Delta P=F_{\text {net }} \Delta t$.

- Newton's third law in typical collision context: Students are asked to determine the features of interaction forces between two objects.

Among the 8 momentum questions, three (Q4, Q9, Q10) involve collisions settings that have zero net force $\left(F_{\text {net }}=0\right)$. Students have extensive practice applying momentum conservation in these contexts during classroom instruction, thus these are considered typical questions. The other 5 questions are considered to involve contexts that are atypical for U.S. students. These questions require students to understand the relation between the change of momentum and impulse due to a nonzero net force applied to the object. It is noted that Q6 and Q7 can also be solved using momentum conservation by treating the two bodies together as an isolated system; however, they are considered atypical since most students are unfamiliar with such contexts.

\section{Research procedure}

The subjects of this study include U.S. college freshmen taking algebra-based introductory mechanics, along with 12th grade Chinese students taking a high school level physics course. Regarding the level of course content, the classical mechanics portion of the Chinese high school physics course is equivalent to that of the algebra-based introductory mechanics course in U.S. colleges. The U.S. students involved in this study are from a large midwestern public university and the Chinese students are from a midranking public high school in a large well-developed coastal city.

The U.S. students were pre- and post-tested, using the assessment previously described, in the beginning and end of the spring semester in 2019. A total of 720 students took the pretest, and 654 students took the post-test. Among these students, 595 took both tests. Meanwhile, 596 Chinese students in grade 12 took the test as post-test at the beginning of the fall semester. For these students, they completed their study of momentum in the spring semester in grade 11, which makes an approximately three-month 
time difference between learning and testing. The pretest and post-test time difference for the U.S. students was approximately 2-3 months. Therefore, both groups had the same amount of time delay between learning and testing, and both groups did not study momentum during this time period.

The primary purpose of this study was to evaluate students' conceptual understanding of momentum, investigate students' knowledge structures, and verify the conceptual framework of momentum. To accomplish the research goals, a number of quantitative and qualitative methods were applied. Exploratory factor analysis was conducted with both the U.S. and Chinese post-test data to identify assessment constructs related to the different context categories. For comparisons of U.S. students between test conditions and question contents, a twoway ANOVA was applied on matched pre-post data to determine possible main and interaction effects. Paired comparisons of student performances were further explored through student $t$ tests. The size of differences between pretest and post-test were measured with Cohen's $d$ effect sizes.

The differences between the U.S. and Chinese students' performances across different question contents were determined using a two-way ANOVA, and further explored through student $t$ tests and Cohen's $d$ effect sizes. As discussed in the analysis section, the Chinese students had achieved a higher level of understanding with more integrated knowledge structures. Therefore, the comparison between Chinese and U.S. students can reveal the influence of different teaching methods on student learning of momentum, while shedding light on features of instruction methods that may promote deep conceptual understanding.

In addition to quantitative data, think-out-loud interviews were conducted with 28 Chinese students randomly selected from the same population pool but who did not take the assessment. The interviews were conducted in a two-week timeframe immediately after the completion of the quantitative assessment. As part of the interview the students were asked to give their responses to each question while explaining their reasoning process. Each interview lasted about $30 \mathrm{~min}$. All interviews were audiotaped and transcribed, which were further analyzed to identify patterns of student reasoning for inferences on students' knowledge structures.

\section{ANALYSIS AND RESULTS}

\section{A. Quantitative study on U.S. students' knowledge structure development}

First, an exploratory factor analysis (EFA) was conducted on the U.S. post-test data, with which a correlation matrix of scores of all items was constructed and eigenvalues were calculated. Because the items were scored dichotomously, the tetrachoric correlation was computed

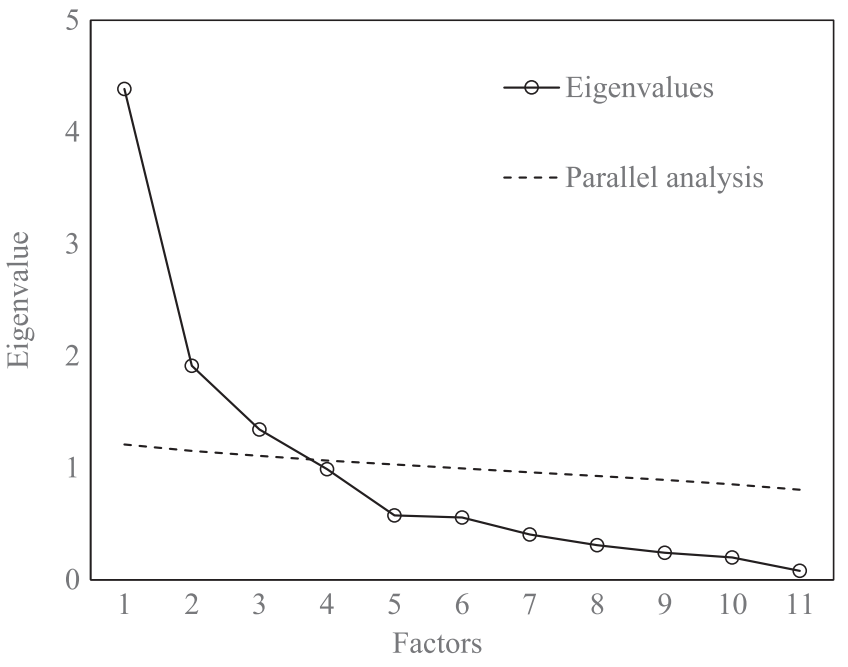

FIG. 2. Scree plot of EFA eigenvalues of the U.S. students' post-test data. The dashed line represents the eigenvalue trend line of the parallel analysis.

instead of the Pearson correlation [29]. To determine the optimal number of factors, the parallel analysis method was used [30]. This process compares the eigenvalues of the actual correlation matrix with those of a correlation matrix of the same dimensions based on random data. A scree plot of the EFA eigenvalues is shown in Fig. 2 along with the eigenvalue trend line of the parallel analysis (the dashed line in Fig. 2). As shown, the first three eigenvalues are above the dashed line indicating a three-factor solution that explains $59 \%$ of cumulative variance (i.e., $22 \%, 18 \%$, and $18 \%$ for factors 1,2 and 3 , respectively). The factor loading of all the questions on these three factors is provided in Fig. 3.

The EFA result confirms that the assessment test contains three categories. These are appropriately matched with the design of three concept domains including momentum conservation, the impulse-momentum theorem, and Newton's third law. As shown in Fig. 3, factor 1

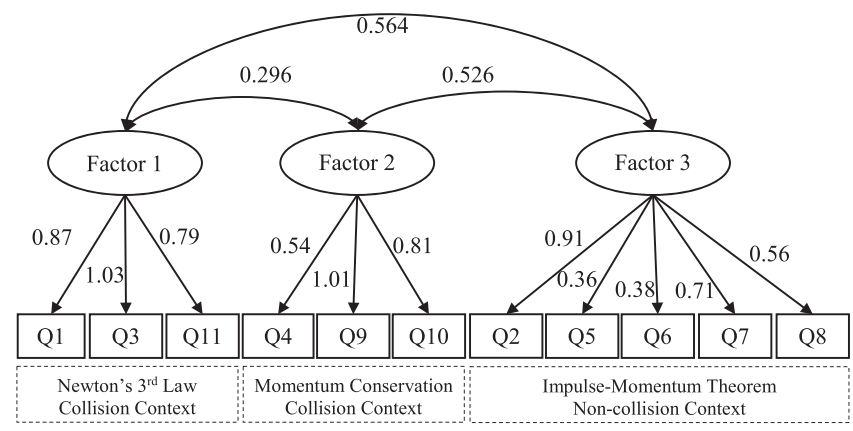

FIG. 3. Factor loadings for EFA of U.S. students' post-test with three-factor solution in which factors 1 to 3 are matched with the assessment design of content and context categories of Newton's third law questions, typical momentum conservation questions, and atypical questions for the impulse-momentum theorem. 
represents Newton's third law, factor 2 represents momentum conservation, and factor 3 represents the impulsemomentum theorem. The results also show that students' responses on the impulse-momentum theorem questions are moderately correlated to their responses on questions involving momentum conservation (0.526) and Newton's third law (0.564). Meanwhile, there is little correlation between students' responses on questions of momentum conservation and Newton's third law, with a correlation coefficient of 0.296 . These results suggest that solving the atypical impulse-momentum questions requires a more extensively connected understanding of the central idea and the related concepts (and thus a more integrated knowledge structure). On the other hand, the students' proficiency in solving momentum conservation problems is less connected to other related conceptual components and may represent a less integrated (or more fragmented) understanding.

Figure 4 shows the U.S. students' pretest and post-test scores. On the pretest, students' scores are approximately $30 \%$ overall and demonstrate small but distinguishable differences among the three concept categories. The post-test results are significantly different between the concept categories and have larger gaps $[F(2,1959)=$ 224.455, $\left.p<0.001, \eta_{p}^{2}=0.186\right]$. The data indicate that students perform best on typical momentum conservation items $\quad\left[t_{\mathrm{MC}, \mathrm{N} 3}(653)=12.476, \quad p<0.001, \quad d=0.634\right.$; $\left.t_{\mathrm{MC}, \mathrm{IM}}(653)=28.889, p<0.001, d=1.337\right]$, followed by Newton's third law items $\left[t_{\mathrm{N} 3, \mathrm{IM}}(653)=10.553\right.$, $p<0.001, d=0.458$ ], with performance on impulsemomentum theorem items the weakest.

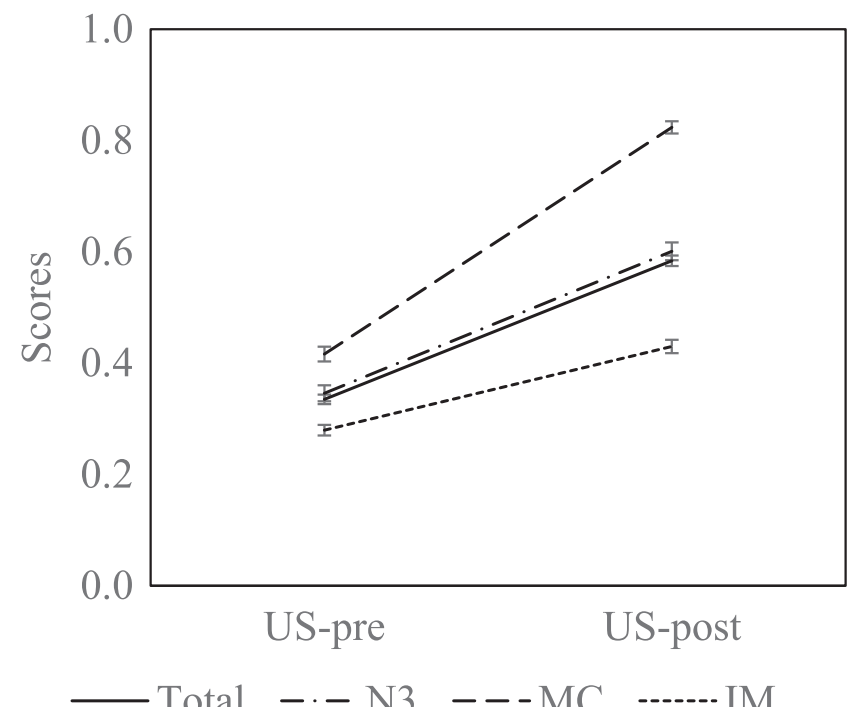

FIG. 4. Pretesting and post-testing performance of students for different concepts including momentum conservation, impulsemomentum theorem, and Newton's third law, as well as the total score. The error bars represent the standard error of the means.
To compare the pretest and post-test changes in different concept categories, a two-way ANOVA was conducted. The results suggest significant interaction among test condition and concept categories $[F(2,4116)=60.483$, $p<0.001, \eta_{p}^{2}=0.029$, with main effects for both question context and test condition (pre or post) $[F(2,4116)=$ 459.049, $p<0.001, \eta_{p}^{2}=0.182 ; F(1,4116)=681.982$, $p<0.001, \eta_{p}^{2}=0.142$, respectively]. This suggests that the current college physics instruction in the U.S. had varying impact on student learning of the three related concepts.

To further evaluate the effectiveness of the instruction, pretest and post-test score gains were also compared. Students had a significant improvement on their overall pre-post score change $[t(595)=19.592, \quad p<0.001$, $d=1.123]$. For the different concept categories, the prepost score improvements are all statistically significant, with the largest improvement observed for the typical collision problems $[t(1372)=23.378, \quad p<0.001$, $d=1.269$ ], followed by the Newton's third law questions $[t(1372)=11.921, p<0.001, d=0.643]$, and with the atypical impulse-momentum questions having the smallest improvement $[t(1372)=9.914, p<0.001, d=0.533]$.

The results discussed above suggest that the instruction in U.S. colleges is effective in training students to solve typical collision problems through the application of momentum conservation equations. However, many students have an underdeveloped conceptual understanding of the impulse-momentum relation. These outcomes are likely caused by the current emphasis in instruction, which often focuses on teaching and practicing collision problems. In comparison, the impulse momentum is only briefly introduced without substantive practices (see details in Appendix B). Students' understanding of Newton's third law also seems to be less developed and weakly connected to their understanding of momentum conservation. Synthesizing these results, it can be inferred that the students' knowledge structures for momentum are in a moderately fragmented transitional stage, with which students are able to apply the momentum conservation equations to limited familiar contexts ( $82 \%$ on post-test), but are unable to make connections to the central idea of the impulse-momentum relation ( $43 \%$ on post-test) and the related Newton's third law concept (60\% on post-test). At this stage, students continue to show signs of novicelike problem-solving behavior with strong reliance on using surface features to identify and match memorized equations and problem-solving procedures.

\section{B. Quantitative study on Chinese students' knowledge structure development}

The assessment outcomes of U.S. students suggest that the current instruction in U.S. colleges often leave students in a transitional stage with fragmented knowledge structures. To explore the influences of instruction on learning 
outcomes, it would be useful to compare the assessment result of U.S. students with that of another population who had gone through a very different style of instruction. Through such comparisons, possible benefits of varying teaching methods can be investigated.

For this part of the study, a Chinese high school population was selected to take the Chinese version of the same assessment but as a post-test. A pretest was not given as the goal was to compare the end results of the accumulated learning outcomes from two very different education environments. The Chinese students learn basic conceptual physics, including force and motion kinematics, as early as 7th and 8th grade. The momentum concept and its relation computations are only formally introduced as part of the high school curriculum, which has clear emphases on both the impulse-momentum theorem and momentum conservation (see details in Appendix A).

Figure 5 shows the post-test scores from the U.S. and Chinese students on the three concept domains. Overall, the Chinese students achieved higher scores than the U.S. students on all three concept domains. A two-way ANOVA shows significant main effects on concept domain and population $\left[F(2,3749)=209.856, p<0.001, \eta_{p}^{2}=0.101\right.$ and $F(1,3749)=543.804, p<0.001, \eta_{p}^{2}=0.127$, respectively]. An interaction effect also exists between concept and population $\left[F(2,3749)=84.979, p<0.001, \eta_{p}^{2}=\right.$ 0.043]. These outcomes indicate that the two populations have different levels of conceptual understanding, which is further demonstrated by $t$-test results showing significant differences in performance between the two populations for three concept domains $\left[t_{\mathrm{N} 3}(1248)=14.453, p<0.001\right.$, $d=0.797 ; t_{\mathrm{MC}}(1248)=3.721, p<0.001, d=0.211$; and $t_{\mathrm{IM}}(1248)=21.729, p<0.001, d=1.230$, respectively].

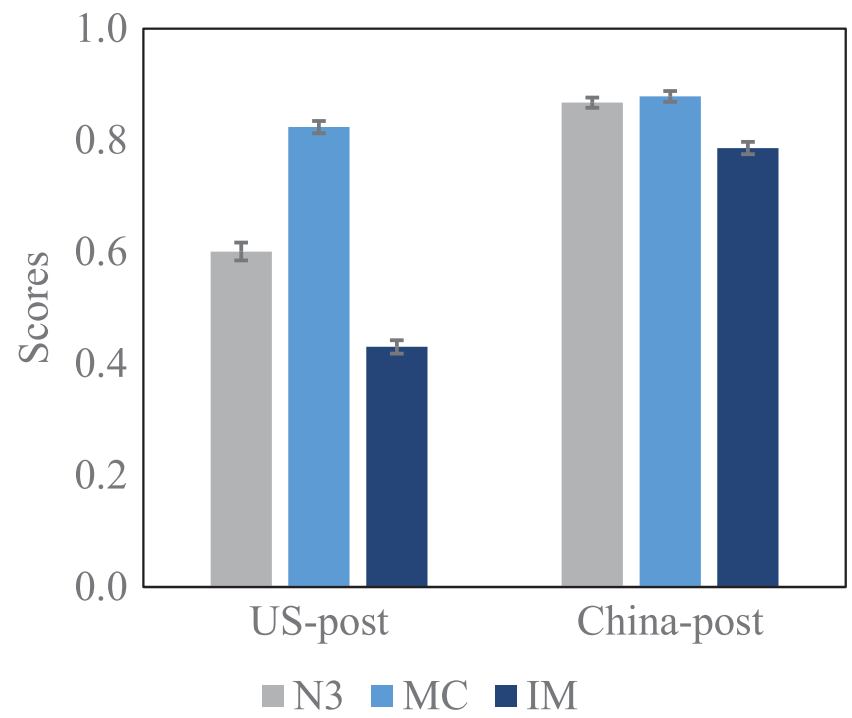

FIG. 5. Post-test performance of U.S. and Chinese students for different concept domains. The error bars represent the standard error of the means.
The difference is the smallest for momentum conservation as both populations did very well $(>82 \%)$ on this concept domain.

Across the three concept domains, both populations had somewhat similar performance patterns. Both scored highest on momentum conservation, lowest on impulsemomentum theorem, and performance for Newton's third law was in the middle. Since the Chinese students have better overall performance with smaller performance gaps among the different concepts than the U.S. students, it suggests that the Chinese students represent a higher level of conceptual development in learning. Comparing the similarity of the performance patterns of the two populations, it appears that momentum conservation is the easiest for students to grasp, which requires the application of conservation equations in limited situations. However, mastering this concept on its own often indicates an intermediate level of understanding with a substantially fragmented knowledge structure (see Fig. 1). In contrast, the impulse-momentum theorem is the hardest for students to develop a good understanding. Therefore, students' performance on impulse momentum can be used as a sign of achieving a deeper understanding integrated around the central idea of the conceptual framework.

The Chinese students' knowledge structures were further explored using factor analysis. The scree plot is illustrated in Fig. 6, which shows a big gap between the first and second eigenvalues. The first eigenvalue also has a large magnitude equivalent to explaining nearly half (44\%) of the variance. These features suggest that students' performance can be sufficiently explained with a prominent single factor, which implies a fairly integrated knowledge structure [see Fig. 7(a) for the loading of the questions on the single factor]. When compared to the factor structure of the U.S. students, which has three distinctive factors, this merging effect further implies that as learning progresses, an

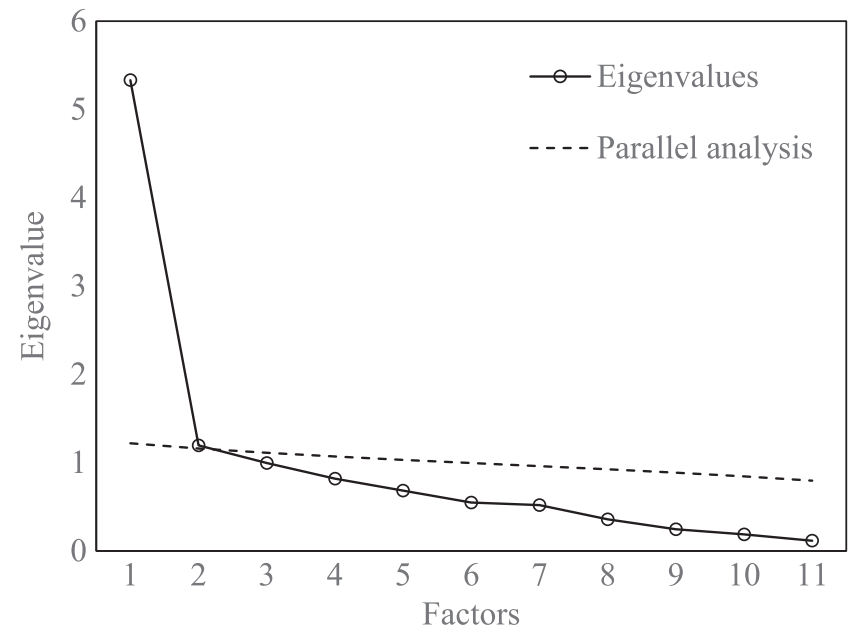

FIG. 6. Scree plot for EFA of Chinese students' post-test with parallel analysis. 

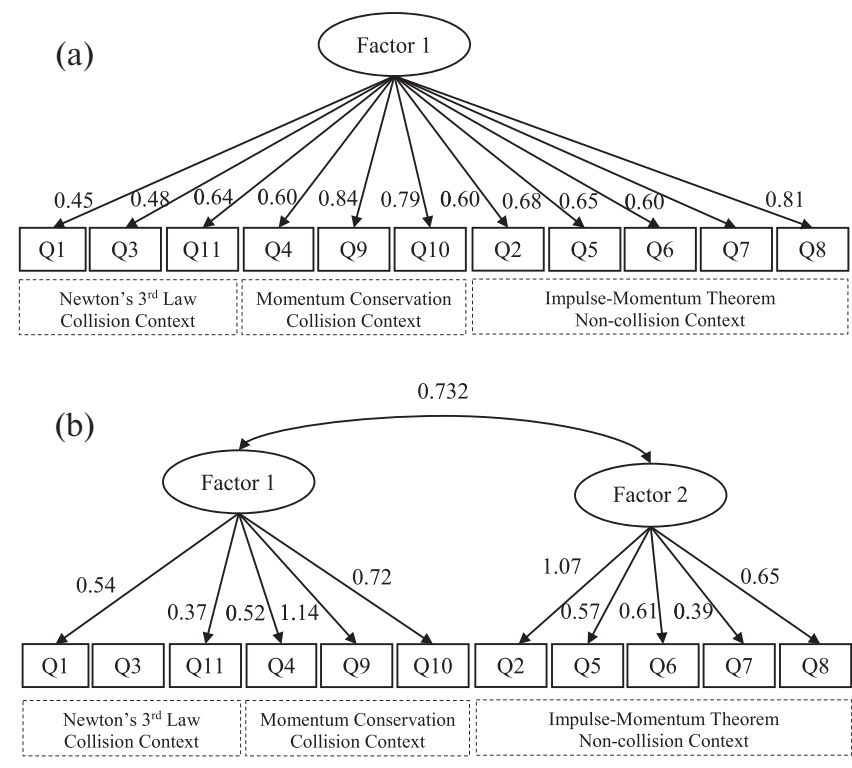

FIG. 7. Factor loadings for EFA of Chinese students' post-test with (a) one factor and (b) two factors.

understanding of Newton's third law (N3) and impulsemomentum theorem (IM) becomes more integrated with momentum conservation (MC), and eventually all three concept domains become integrated into a single wellconnected knowledge structure.

In order to study finer details of students' knowledge structures, it is informative to explore the second factor, which is on the border just above the trend line of the parallel analysis. The two-factor solution explains a total of $51 \%$ cumulative variance, which is only slightly increased from the one-factor solution. The loadings of the questions on the two-factor solution are illustrated in Fig. 7(b). The results show that the two factors are highly correlated, which is consistent with the single-factor solution. The unique feature represented by the two-factor structure is the effect of question context. As shown in Fig. 7(b), questions with collision contexts are mostly loaded on factor 1 (except for Q3, which is not loaded on either of the factors), while questions with noncollision contexts are all loaded on factor 2 . The results suggest that besides the main effect from conceptual understanding, the question context also makes moderate secondary influence on students' performance. It appears that even though the Chinese students have developed more integrated knowledge structures, their learning still reveals signs of context dependence [31]. Therefore, further progression of knowledge integration requires more comprehensive development, reorganization, and synthesis of the connections among the contextual features, conceptual components, and central idea, in order to form a well-integrated network of relations and variables.

As a means of investigating the differences in learning between understanding of momentum conservation and the

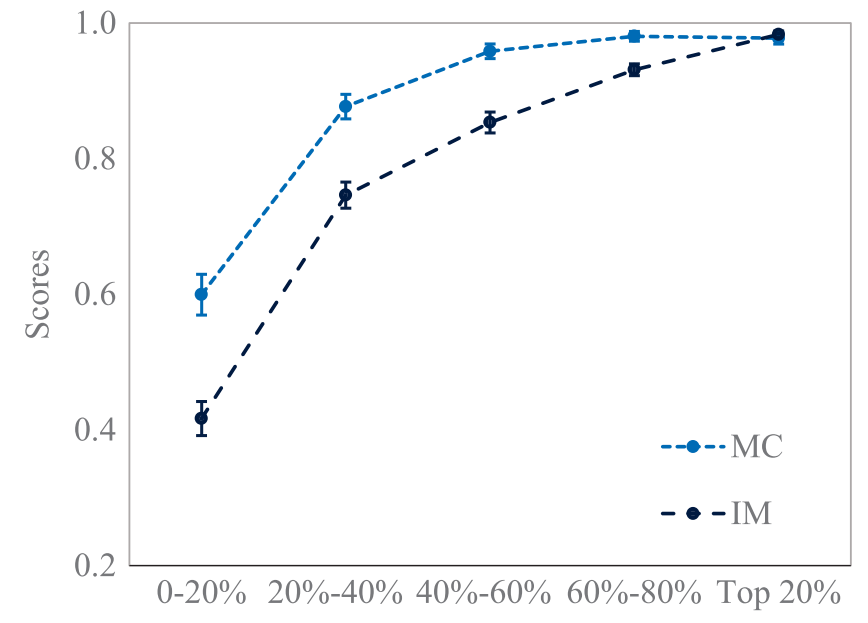

FIG. 8. Scores on momentum conservation and impulsemomentum theorem of Chinese students in different performance groups. The five performance groups are determined by students' physics exam scores. The error bars represent standard error.

impulse-momentum theorem among students of different performance levels, the Chinese students were assigned to five performance groups that each contain $20 \%$ of the total sample. The performance level is determined using students' exam scores in physics, which reflect their overall learning of mechanics. The results are illustrated in Fig. 8, which shows that the scores on impulse-momentum theorem are consistently below the scores on momentum conservation for all groups. The low to medium performance groups $(0 \%-60 \%)$ have more pronounced performance gaps between the two concepts than the groups at higher performance levels $(60 \%-100 \%)$. This confirms that the impulse-momentum theorem is typically harder to learn than momentum conservation, and it becomes better developed only when students approach expertlike understanding.

\section{Qualitative study of Chinese students' reasoning process}

In order to identify students' possible reasoning pathways and their knowledge structures, 28 Chinese students were randomly selected from the same population pool to participate in think-out-loud interviews using the same assessment questions. Note that these students did not take the post-test. As part of the interview, students were asked to solve the questions while explaining in detail how they arrived at their answers. Based on students' responses, three levels of students' understanding were identified and described below.

Novice level: These students solve problems relying on memorization of equations and with little reasoning. The contextual variables and features cue their memory of related equations without meaningful reasoning connecting to other related conceptual components and ideas. The knowledge structure is largely fragmented with only local 
connections that link contextual variables directly to equations. Therefore, novices can only solve a limited number of familiar problems, which had been practiced and memorized from prior coursework. Interview excerpts from students who exhibit this type of problem-solving behavior are given below:

Student A: (Response to question 2) "I think they have the same momentum... because, well, the formula is $P=F \quad \ldots$ oh, it's $P=F t$, right? Is it $P=F t$ ?" (Response to question 4) "This is not very clear. I feel like that Imemorized a momentum formula before, which is about calculation after two objects collide...but the state of Block B after the collision is unknown here."

Student B: (Response to question 5) "Oh, the momentum equals mass times velocity, right? I saw the unit in the answers; it seems to be correct. So, in this question, mass is known, and velocity... and velocity may be calculated with some formulas, right? Then we can get the answer of the momentum by multiplying mass with velocity."

These students, like most typical novices, could only answer questions by matching contextual features and variables with memorized equations. For instance, student A noticed that the two variables of force and time were given in question 2, and it prompted him to vaguely recall the equation $P=F t$ but without understanding the conceptual unpinning. It is clear that the connection the student made between the given variables and the possible outcomes was on the surface level to match variables with equations with little conceptual understanding. In addition, student A recognized that question 4 is a collision problem and the student tried to relate it to other collision problems from memory. However, the contextual differences between the given question and the memorized example, such as whether certain blocks are stationary or moving, made the memorized case not directly applicable to the given situation, and therefore the problem was not correctly solved.

Meanwhile, student $\mathrm{B}$ recalled the common defining equation of momentum $(P=m v)$ cued by some variables given in question 5. This student then tried to find all the needed variables in the question to solve for the answer. However, this question is best solved with the impulsemomentum theorem $(\Delta P=F \Delta t)$. Using the equation of $P=m v$, one would need to determine the change of velocity through kinematic relations, which is computationally more complex. As a result, the student was unable to correctly solve the problem.

The behaviors the two students demonstrated here support the idea illustrated in the conceptual framework of momentum that novices often have a fragmented knowledge structure with local connections among surface features, leading them to rely on contextual variables as the primary resources to construct problem-solving pathways.
Transitional level: these students demonstrate sufficient reasoning in applying momentum conservation in typical collision situations, but have significant difficulties in understanding and applying the impulse-momentum theorem for problem solving. The students in the study seemed to predominantly focus on the momentum conservation concept, and would often get confused on problems related to the impulse-momentum theorem in non-collision contexts. For instance, student $\mathrm{C}$ successfully solved question 4 (momentum conservation in collision context), but had trouble with question 2 (impulse-momentum theorem in noncollision context):

Student C: (Response to question 2) "This one... I cannot deal with this question. I think it is about the impulse-momentum theorem, I remembered that is $F t=m \Delta v$, the momentum is changing, and then I don't know how to do it. (Responses to question 4) "The correct answer is "c," just use momentum conservation, that means the initial momentum of block $A$ equals to the final momentum of block $A$ and block $B$, then you made it."

Among the transitional students, some appeared to have more developed understanding than others. However, even the better performing students still do not have an expertlike knowledge structure integrated around the central idea of the impulse-momentum theorem and their problemsolving strategies relied primarily on applying $p=m v$. They were often able to perform valid reasoning by using kinematic relations to evaluate the unknown variable, the velocity, based on the given parameters (e.g., force, mass, and time). These students had a moderate level of success in solving impulse-momentum theorem questions due to reasoning pathways which involved using kinematic relations to obtain velocity, a process that is often convoluted and computationally challenging. The following two students' responses demonstrate this finding:

Student D: (Response to question 5) "This problem should also use momentum conservation. Because the block $A$ is moving to the right on the board, the board is subject to a force of friction to the right; then the board starts to move to the right... then one second later, the velocity of the board can be calculated, and the speed of block A can also be calculated. So we can get all the final velocities of both block and board. Then we can figure it out by taking the final momentum of block $A$ minus its initial momentum." (The student didn't get the correct numerical answer.)

Student E: (Response to question 5) "In this situation, block A decelerates and slows down to $3 \mathrm{~m} / \mathrm{s}$. Suppose the board doesn't move here, then the change of momentum of block $A$ can be calculated by $m\left(v_{\text {finial }}-v_{\text {initial }}\right)$... so it is 20. The answer is A." (The correct answer is f.) 
Students similar to these two had a good understanding of momentum conservation, but they hadn't developed an understanding of the impulse-momentum theorem. Their knowledge structures were partially fragmented, with limited and isolated pathways connecting only momentum conservation with familiar contexts. As stated in the conceptual framework of momentum, their conceptual understanding and problem-solving strategies were still context dependent, and the connections were often constructed between familiar contextual variables and momentum conservation. Students had not been able to develop a good understanding of the central idea of impulsemomentum theorem and make meaningful connections to link impulse-momentum theorem, momentum conservation, and the varieties of problem settings and variables.

Expertlike level: The most developed students were found to approach the expert level of understanding. They were able to solve both the momentum conservation and the impulse-momentum theorem problems using multiple strategies. For question 7 in particular, these students could reason through the scenario by either considering the two people as two separate point masses, or as a system as a whole. If the former scenario is considered, then according to Newton's third law the two forces exerted on each other are equal in magnitude and opposite in direction. Consequently, through using the impulse-momentum theorem, the change in momentum should be identical. If the two people are considered to be a system as a whole, there should be no applied external force, i.e., the only interactions that occur in this scenario are the internal forces within the system. Therefore, the momentum of the system is conserved, which means that the change in momentum of the two bodies should be identical. The following responses of expertlike level students demonstrate these two ideas.

Student F: (Response to question 7) "This is A. The external net force in the vertical direction of each person is zero and there were only interactions from each other in the horizontal direction. According to Newton's third law, the force applied on these two persons are equal and lasts for the same time, and the initial momentum of each one is zero. So the magnitude of their momentum is the same but in opposite directions."

Student G: (Response to question 7) "Ignore the friction, the total momentum of the system of these two persons should be conserved, because there's only interactions between them. Then we can know that the sum of Amy's momentum and Jane's momentum should be 0 all the time. Therefore, A is the right answer, the magnitude equals while the directions of the two are opposite to each other."

These students appeared to have developed a deep understanding of the central idea and were able to correctly apply the idea to answer most of the questions. These students recognized the possible connections among the concept components and various contextual features. In their knowledge structure, the impulse-momentum theorem and momentum conservation concept appear to be meaningfully linked and unified as a single body of understanding. The following excerpts show how these students solve related problems in general by using an integrated understanding of the concept.

Student F: "To see if it can form a system without external forces, if it does, I can probably use momentum conservation, otherwise use the impulse-momentum theorem. Sometimes, you have to combine them."

Student G: "Use Ft when you know force and time, and use $m v$ when mass and velocity is known. However, there needs to be a specific analysis. The conservation of momentum... first of all, as a result of an experiment, it is only true when the whole system is free of external forces or when the external forces sum to zero. And the change in momentum is equal to $F t$, here $F$ means the net force on the object, and there is a correspondence between force and time. Besides, momentum is a vector."

As shown in these excerpts, the students exhibit expertlike problem solving behaviors. They started by analyzing the forces within the system, and then determined their approaches. They made the necessary connection that the change in momentum is equal to $F_{\text {net }} \Delta t$, and when $F_{\text {net }}=0$, then momentum is conserved. Through this connection, they were able to solve the given problems. This type of behavior is an obvious contrast to that exhibited by novicelike students who tend to use a problem-solving strategy that relies on matching the given contextual parameters and variables with memorized approaches and equations.

Among all interviewed students, the number at each level included 13 experts, 10 transitional, and 5 novices. Their performance on each set of items is shown in Table II. Because of the small sample sizes, the results are for information purposes only without any statistical implications.

In general, since many Chinese students had achieved high scores within all concept categories, they were designated as approaching the expert level of understanding with more integrated knowledge structures. Therefore, the one-factor EFA outcome is consistent with the students'

TABLE II. The average performance of interviewed students at different levels.

\begin{tabular}{lcccc}
\hline \hline Level & Total & N3 & MC & IM \\
\hline Novice & $29.09 \%$ & $33.33 \%$ & $20.00 \%$ & $32.00 \%$ \\
Transitional & $71.82 \%$ & $93.33 \%$ & $86.67 \%$ & $50.00 \%$ \\
Expertlike & $100 \%$ & $100 \%$ & $100 \%$ & $100 \%$ \\
\hline \hline
\end{tabular}


quantitative data and interview outcomes. Putting these together, it is evident that novicelike students operated with fragmented knowledge structures and therefore were only able to solve momentum conservation questions in familiar contexts using memorized equations. The transitional students had more developed understanding about momentum conservation and could solve most momentum conservation questions. However, these students still lacked the understanding of and connection to the central idea of the impulse-momentum theorem. As a result, they were only able to solve limited cases of the impulse-momentum theorem questions. Although their knowledge structures were more integrated than the novice students, they were still missing important links to the central idea. The expertlike students had achieved well-integrated knowledge structures and had developed problem-solving approaches of applying the central idea in scenarios of $F_{\text {net }}=0$ or $F_{\text {net }} \neq 0$ such that they could correctly and effectively solve problems.

\section{CONCLUSION}

In this study, a conceptual framework of momentum was developed and used to guide the assessment of student knowledge structures in learning momentum concepts. Based on the qualitative and quantitative analysis of the assessment outcomes, students' understanding fell into three progression levels of knowledge integration including novicelike, transitional, and expert-like.

The novicelike students struggled with most of the questions, and their understanding appeared to be closely tied to surface features of the problems without deeper connections to the central idea. They seemed to have memorized aspects of the concepts in fragments, which appeared to be directly tied to the contextual features and variables. Consequently, they were unable to provide meaningful reasoning or explanations to their answers and were only able to answer a few questions that could be easily matched to previously memorized examples and equations.

The students in the transitional level were able to answer most or all of the momentum conservation questions, however, they had significant difficulties with questions involving the impulse-momentum theorem. Their knowledge structures seemed to be locally connected among conceptual components and contextual variables on the momentum conservation side, with few connections made to the central idea of the impulse-momentum theorem. As a result, their problem-solving strategies were context dependent and biased towards momentum conservation in familiar contexts.

The expertlike students were able to answer most or all of the questions by appropriately applying the concept of momentum conservation and the impulse-momentum theorem. They appear to have successfully developed their understanding around the central idea with well-established connections among most key variables and relations, forming an integrated knowledge structure. Therefore, when solving problems, these students were able to focus on the central idea and use it to guide their problem-solving strategies without being tied to the surface features of the problem contexts.

The problem-solving behaviors of students at the different levels reveal a progression from surface to deep understanding, which develops from accumulation of information on surface features, to construction of more networked knowledge structure, and finally to the fully integrated understanding. Through the progression of knowledge integration, students demonstrate improved accuracy on problem-solving tasks, reduced context dependency, and more explicit use of the central idea. All of these are indications that the students are approaching the expert level in conceptual understanding and problem solving.

As demonstrated in the two tracks of previous studies $[19,20]$, being able to identify the central idea is essential to knowledge integration and deep understanding. For the topic of momentum, students must recognize that the definition of momentum as a function of mass and velocity is not an arbitrary choice; it arises from the impulsemomentum theorem that makes the connection between the change in momentum and the impulse applied on an object [22]. It is for this reason that the impulse-momentum theorem is chosen to be at the core of the central idea. Comparing the instruction between the two countries, the Chinese curriculum places equal emphasis on momentum conservation and the impulse-momentum theorem, while the U.S. curriculum places greater emphasis on momentum conservation. The results of this study, which show that the Chinese students had gained a deeper understanding than the U.S. students due to their better performance on the assessment questions, suggests that the emphasis on the central idea of the impulsemomentum theorem can be an essential instructional strategy to help students make the necessary connections within their knowledge structure leading to a deeper conceptual understanding of momentum.

In conclusion, this study extends previous work on the use of conceptual frameworks into the topic of momentum. The results show that instruction with a primary emphasis on momentum conservation can leave students in the transitional stage with fragmented knowledge structures. In order to help students develop integrated knowledge structures and deeper understanding, the impulsemomentum theorem is an important central idea that needs to be emphasized in instruction. Contrasting the assessment results on impulse-momentum theorem and momentum conservation can provide a way to measure students' knowledge integration and level of understanding for the topic of momentum. This study further demonstrates that the conceptual framework approach is effective in modeling and analyzing knowledge integration in learning 
physics and can also provide valuable guidance on developing instructional methods that promote deep learning.

\section{ACKNOWLEDGMENTS}

The research is supported in part by the National Science Foundation Grants No. DUE-1431908 and No. DUE1712238, and by the East China Normal University Scholarship Program for Graduate Students' Short-term Overseas Research Scholarship (No. 20180620048). Any opinions, findings, and conclusions or recommendations expressed in this paper are those of the authors and do not necessarily reflect the views of the funding agencies.

\section{APPENDIX A: THE INSTRUCTION OF MOMENTUM IN CHINESE HIGH SCHOOLS}

The instruction is delivered in five traditional lectures that each last $45 \mathrm{~min}$ (see Fig. 9). The curriculum is designed based on the national standard enforced to be followed by all high schools in China. In the first lesson, the teacher leads the students to perform a series of collision experiments to find that the product of mass and velocity is conserved in a collision process, and then defined it as the concept of momentum.

In the second lesson, the teacher asks the students to analyze the situation where the velocity of a single object changes from $v_{1}$ to $v_{2}$ after a constant force $F$ is applied to it for a certain time $\Delta t$, and to find out the relationship between these variables. Students try to use Newton's laws, which they have already learned before, to come up with relevant equations: $F=m a=m\left(v_{2}-v_{1}\right) / \Delta t$. Then, they will get $F \Delta t=m\left(v_{2}-v_{1}\right)$, which is impulse-momentum theorem. Afterwards, students practice solving a variety of problems in related contexts, which aims to help students getting familiar with the relationship between the force, time and the change in momentum.

In the third lesson, the teacher guides the students to rethink the problem of collision of two balls from the perspective of Newton's law of motion. They discuss the interactions between the colliding balls, and eventually get the same conclusion as from the experiments, which is $m_{1} v_{1}+m_{2} v_{2}=m_{1} v_{1}^{\prime}+m_{2} v_{2}^{\prime}$. Furthermore, the condition of momentum conservation is explained: the net force of the system is zero.

In the fourth and fifth lessons, the teacher guides the students to solve one-dimensional collision problems, including elastic collision and inelastic collision, and recoil motion (explosion). The law of momentum conservation is practiced in analyzing these problems.

In general, the teacher focuses on guiding the students to solve a variety of problems in different situations using the law of conservation of momentum as well as the impulsemomentum theorem. In addition, most schools usually spend less time on doing related experiments and demos, but focus on the formal discussion of impulse-momentum

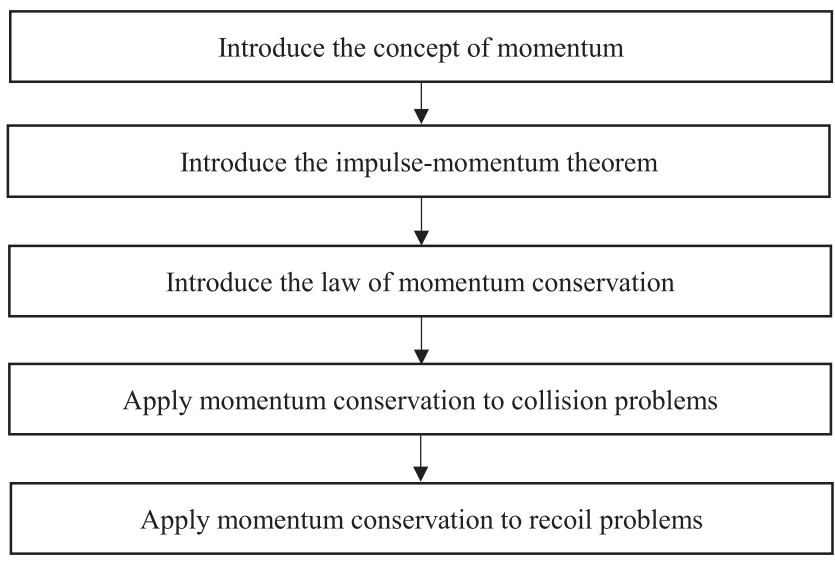

FIG. 9. The process of instruction on momentum in Chinese high schools.

theorem and the conditions of momentum conservation. In the latest curriculum standard, the requirement for understanding of the impulse-momentum theorem is even more explicitly emphasized than before, while the law of momentum conservation still remains as a key requirement.

\section{APPENDIX B: THE INSTRUCTION OF MOMENTUM IN THE U.S. UNIVERSITY IN THIS STUDY}

The instruction is typically delivered in three traditional lectures that each last about $55 \mathrm{~min}$ (see Fig. 10). The three lectures include an introduction to the concept of momentum and momentum conservation, discussion of different types of collision problems, and discussion of the momentum problems of multiparticle systems. In the introduction of the concept of momentum, the impulse-momentum theorem is typically discussed for less than $1 / 3$ of the lecture time (about 15-20 min), while the momentum conservation is often the heavily emphasized concept taught with demos and simulations. Momentum conservation is further enhanced in the following two lectures with practices of various kinds of collisions and systems, including elastic collisions, inelastic collisions, one-dimensional collisions, two-dimensional collisions, and so on.

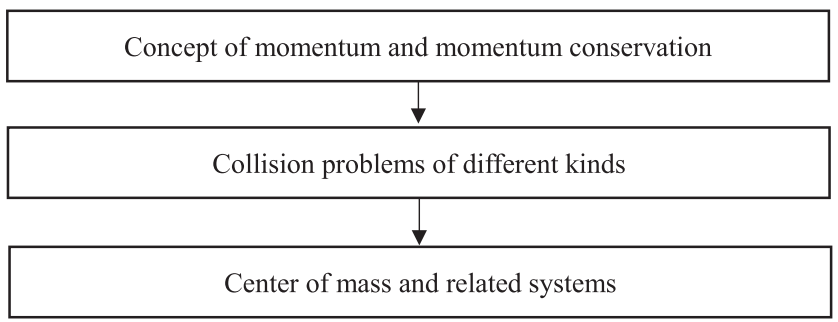

FIG. 10. The process of instruction on momentum in the U.S. university in this study. 


\section{APPENDIX C: ASSESSMENT TEST OF MOMENTUM}

The assessment test of momentum includes 11 questions summarized in Table III. The full test is given in the Supplemental Material [32].

TABLE III. Summary of assessment questions on momentum.

\begin{tabular}{lllc}
\hline \hline Item & \multicolumn{1}{c}{ Content } & \multicolumn{1}{c}{ Context } & Key \\
\hline Q1 & Newton's third law (N3) & Collision & E \\
Q2 & Impulse-momentum theorem (IM) & Applied force & B \\
Q3 & Newton's third law (N3) & Collision & F \\
Q4 & Momentum conservation (MC) & Collision & C \\
Q5 & Impulse-momentum theorem (IM) & Friction & F \\
Q6 & Impulse-momentum theorem (IM) & Friction & A \\
Q7 & Impulse-momentum theorem (IM) & Explosion & A \\
Q8 & Impulse-momentum theorem (IM) & Applied force & B \\
Q9 & Momentum conservation (MC) & Collision & C \\
Q10 & Momentum conservation (MC) & Collision & D \\
Q11 & Newton's third law (N3) & Collision & F \\
\hline \hline
\end{tabular}

[1] M. H. Chiu, Algorithmic problem solving and conceptual understanding of chemistry by students at a local high school in Taiwan, Proc. Natl. Sci. Council Republic of China Part D, Math. Sci. Technol. Education 11, 20 (2001).

[2] M. H. Chiu, C. Guo, and D. F. Treagust, Assessing students' conceptual understanding in science: An introduction about a national project in Taiwan, Int. J. Sci. Educ. 29, 379 (2007).

[3] E. Kim and S. J. Pak, Students do not overcome conceptual difficulties after solving 1000 traditional problems, Am. J. Phys. 70, 759 (2002).

[4] S. C. Nurrenbern and M. Pickering, Concept learning versus problem solving: Is there a difference?, J. Chem. Educ. 64, 508 (1987).

[5] L. Bao and K. Koenig, Physics education research for 21st century learning, Disciplinary Interdisciplinary Sci. Educ. Res. 1, 1 (2019).

[6] M. Alonso, Problem solving vs. conceptual understanding, Am. J. Phys. 60, 777 (1992).

[7] A. B. Champagne, R. F. Gunstone, and L. E. Klopfer, A perspective on the differences between expert and novice performance in solving physics problems, Res. Sci. Educ. 12, 71 (1982).

[8] M. T. Chi, P. J. Feltovich, and R. Glaser, Categorization and representation of physics problems by experts and novices, Cogn. Sci. 5, 121 (1981).

[9] J. Larkin, J. McDermott, D. P. Simon, and H. A. Simon, Expert and novice performance in solving physics problems, Science 208, 1335 (1980).

[10] W. J. Gerace, R. J. Dufresne, W. J. Leonard, and J. P. Mestre, Problem Solving and Conceptual Understanding, in Proceedings of the 2001 Physics Education Research
Conference, Rochester, NY, edited by K. Cummings, S. Franklin, and J. Marx (AIP, New York, 2001).

[11] B. S. Eylon and F. Reif, Effects of knowledge organization on task performance, Cognit. Instr. 1, 5 (1984).

[12] A. H. Schoenfeld and D. J. Herrmann, Problem perception and knowledge structure in expert and novice mathematical problem solvers, J. Exper. Psychol. Learn. Memory Cogn. 8, 484 (1982).

[13] J. L. Snyder, An investigation of the knowledge structures of experts, intermediates and novices in physics, Int. J. Sci. Educ. 22, 979 (2000).

[14] H. S. Lee, O. L. Liu, and M. C. Linn, Validating measurement of knowledge integration in science using multiplechoice and explanation items, Appl. Meas. Educ. 24, 115 (2011).

[15] M. C. Linn, The knowledge integration perspective on learning and instruction, in The Cambridge Handbook of the Learning Sciences (Cambridge University Press, New York, 2005), pp. 243-264.

[16] J. Shen, O. L. Liu, and H. Y. Chang, Assessing students' deep conceptual understanding in physical sciences: an example on sinking and floating, Int. J. Sci. Math. Educ. 15, 57 (2017).

[17] M. Kubsch, J. Nordine, K. Neumann, D. Fortus, and J. Krajcik, Measuring integrated knowledge-a network analytical approach, in Proceedings of the 13th International Conference of the Learning Sciences (ICLS) (ICLS, London, UK, 2018).

[18] J. Nordine, J. Krajcik, and D. Fortus, Transforming energy instruction in middle school to support integrated understanding and future learning, Sci. Educ. 95, 670 (2011). 
[19] Y. Nie, Y. Xiao, J. C. Fritchman, Q. Liu, J. Han, J. Xiong, and L. Bao, Teaching towards knowledge integration in learning force and motion, Int. J. Sci. Educ. 41, 2271 (2019).

[20] R. Dai, J. C. Fritchman, Q. Liu, Y. Xiao, and L. Bao, Assessment of student understanding on light interference, Phys. Rev. Phys. Educ. Res. 15, 020134 (2019).

[21] C. Singh and D. Rosengrant, Multiple-choice test of energy and momentum concepts, Am. J. Phys. 71, 607 (2003).

[22] R. A. Lawson and L. C. McDermott, Student understanding of the work-energy and impulse-momentum theorems, Am. J. Phys. 55, 811 (1987).

[23] R. J. Raven, The development of the concept of momentum in primary school children, J. Res. Sci. Teach. 5, 216 (1967).

[24] G. C. Rosa, C. Cari, N. S. Aminah, and J. Handhika, Students' understanding level and scientific literacy competencies related to momentum and impulse, J. Phys. Conf. Ser. 1097, 012019 (2018).

[25] B. G. Dega and N. Govender, Assessment of students' scientific and alternative conceptions of energy and momentum using concentration analysis, African J. Res. Math. Sci. Technol. Educ. 20, 201 (2016).

[26] T. G. K. Bryce and K. MacMillan, Momentum and kinetic energy: Confusable concepts in secondary school physics, J. Res. Sci. Teach. 46, 739 (2009).

[27] E. Mazur, Principles \& Practice of Physics (Pearson, Upper Saddle River, NJ, 2015).

[28] L. Bao, K. Hogg, and D. Zollman, Model analysis of fine structures of student models: An example with Newton's third law, Am. J. Phys. 70, 766 (2002).

[29] C. Hofacker and B. Muthén, Testing the assumptions underlying tetrachoric correlations, Psychometrika 53, 563 (1988).

[30] J. Hayton, D. Allen, and V. Scarpello, Factor retention decisions in exploratory factor analysis: A tutorial on parallel analysis, Organ. Res. Meth. 7, 191 (2004).

[31] L. Bao and E. F. Redish, Model Analysis: Representing and assessing the dynamics of student learning, Phys. Rev. ST Phys. Educ. Res. 2, 010103 (2006).

[32] See Supplemental Material at http://link.aps.org/ supplemental/10.1103/PhysRevPhysEducRes.16.010130 for the full version of Assessment Test of Momentum. 